\title{
Research on the Fast Condition Assessment Method of Bridge Based on the Simple Monitoring System
}

\author{
Xu Yichao ${ }^{1,2, a}$, Wang Cuili ${ }^{3}$, Yu Xuerong ${ }^{3}$, Rao Kang ${ }^{3}$, Zhao Chongde ${ }^{3}$ \\ ${ }^{1}$ The State Key Laboratory on Safety and Health of In-service Long-span Bridges, Nanjing China \\ ${ }^{2}$ Jiangsu Transportation Institute Co., Ltd. Nanjing China \\ ${ }^{3}$ Fuzhou Changle highway bureau, Fuzhou China \\ axyc576@jsti.com
}

Keywords: simple monitoring system, condition assessment, quasi-static load testing, displacement influence line

Abstract. In this paper, after the establishment of a simple monitoring system, a multithread wireless acquisition system is developed to facilitate the acquirement, in a quasi-static loading test, of the displacement influence line of the control points in the girder of a bridge. Loading test outcomes are then inferred directly from the quasi-static loading test. Corresponding preliminary verification is implemented on a bridge locating in a provincial artery of some state. It is indicated in the results that this study has a high applicability as fast loading tests and in condition assessment researches. And then manifested are its theoretical significance and engineering practicality.

\section{Introduction}

In recent years, increasingly higher requirements are emerging on road transport together with the fast growth of the economy of China. As the highway construction and highway bridge construction prospers in China, more than 700,000 bridges of all kinds has been erected. In terms of project quantity, construction scale, or technical difficulty, China has now been one of the bridge construction powers in the world. Predication has been made that till the year 2020 or so, there will be more than 100,000 bridges built in China. However, operation safety raises as a aggravating problem in China. The newest statistical data disclosed that, among the existing bridges about 90,000 bridges are of technical condition class D or E, that is, dangerous. And the number of dangerous bridges is still climbing up though some counteracting measures are being taken.

Since 1950s, it has been heeded and stressed the structural damage monitoring and the condition assessment of existing bridges. Massive literature, together with some published methodologies and guidelines, could be found. Quantitative analysis on bridge structural condition has been proved hard in general conditions due to various factors, e.g. the design load class, manufacturing method and quality, the performance of involved material and the condition of bridge. It is then very unpractical attempting to know the accurate bearing capacity of a bridge.

Both domestic and foreign scholars have paid their efforts to propose assessment method for bridge structural condition in these years. Some are relatively more mature and receive wide application; while some are still in their research stage and need more real cases to prove their validity. All these methods, generally speaking, consist of appearance inspection, calculation check and loading test. It should be noted that, though appearance inspection and calculation check could be quick assessment methods for bridge structural condition, subjectivity accompanies the assessment process while accuracy needs further improvement. As for loading test, despite of its agreeable accuracy, its disadvantages, i.e. time-consuming, labor-consuming and long-time traffic shutdown are not good. Therefore, it is necessary to propose a fast carrying capacity assessment method with high accuracy and high speed.

\section{Existing fast condition assessment of bridge}

Along with the progress of modern electronic techniques and structural finite element analysis, test method has migrated from simple strain gauge test to test with micro-processor controlled data 
acquisition, and to the hybrid test method that combines analytical model and field data. BDI wireless structural test system is a low-frequency quasi-static test system based on such hybrid test method, providing an integrated method for structural durability evaluation method and detection technique. The quasi-static loading test determines influence lines under vehicle loading, compares the measured influence line with the calculated influence line, and could adjust bridge model parameters to make them consistent.

In 2006, China Academy of Transportation Sciences completed a project and published the draft Specification for Inspection and Evaluation of Load-bearing Capacity of Highway Bridges, which elaborates and adjusts the in-force standard Evaluation Methods of Load-Carrying Capacity of Existing Highway Bridges (Trial). The draft became in force in 2011 after authorized by Ministry of Transport of People's Republic of China as JTG/T J21-2011. On basis of this specifications, many exploration and practices have been carried out. Zhou et al [1] in 2006 studied the difference between the theoretical influence line and the actual influence line, taking beam-type bridge as studying object, to derive an iterative formula. Thereafter iterative scheme is employed to evaluate actual carrying capacity of bridge. Features of such technique were unveiled by confidence test, e.g. reliability, intuition and economy. In 2009, Li [2] and Liao et al [3], further verified the feasibility of evaluating the carrying capacity of short and middle span bridge based on influence line, by a static loading test on the Wuyang bridge in Zhong County, Chongqing. Qualitative judgments on computational model of complex structures, such as arch bridges or dangerous, based on actual influence line, could reduce possible risk of static loading test by improving the reliability of loading scheme. In the same year, Zhang et al [4] proposed a quasi-static loading test-based fast condition assessment method, which actual influence line-based model calibration techniques are used for determining the carrying capacity of bridges. This method in contrast to calculation checking is with less subjectivity and higher accuracy, while compared to normal loading test, lower expenditure and less shut-down time (it can reduce the shutdown time from more than 8 hours to with half an hour.). The method could serve as a substitute in some cases of normal loading test, or an intermediate method between the calculation checking and the conventional loading test method. Afterwards, Zhang' team of Southeast University implemented a study on the distributed FBG sense technique-based fast loading test and condition assessment of beam-type bridge, proposed a calculation method of the influence line of controlling point using measured strain field of the full bridge, and further discussed the analytical algorithm to derive directly loading test results by the help of the quasi-static test results other than complex model calibration. The difficulty of data processing of the quasi-static loading test is then mitigated.

\section{Fast Condition Assessment Method of Bridge Based on the Simple Monitoring System}

In comparison with traditional static loading test method, quasi-static loading test method is a fast condition assessment method of bridge structure based on model calibration and the comparative analysis of influence lines. The two aforementioned fast condition assessment methods are all grounded on static loading test, from the perspective of model calibration and distributed sensor, respectively. Tests have proved their feasibility in specific cases. However, it is the traditional strain measuring technique that the quasi-static loading test-based fast condition assessment method adopts, which inevitably results in low accuracy, due to the uniform damage assumption and the single point strain-measurement. Similarly, in the distributed FBG sense technique-based fast loading test and condition assessment of beam-type bridge, the problem of low accuracy also exists but due to the necessary transformation between different physical quantities. Problems, such as high test expenditure and long test preparation time owing to sensors distribution along full-bridge, are also drawbacks of this method.

Therefore, after careful scrutiny on available relative techniques, e.g. sense technique, data acquisition and transmission technique, an inclinometer-based multi-point simultaneous dynamic bridge deflection measurement method. In terms of sense equipment, a high-precision bi-axial inclinometer was developed jointly by us and Wuxi Bewis Sensing Technology LLC. The 
inclinometer has a resolution of $0.0005^{\circ}$ and a precision of $0.001^{\circ}$. Massive laboratory and site tests have disclosed its high measurement precision $(0.1 \mathrm{~mm})$, interruption resistance and capability to real-time sensing. Temperature and visibility become negligible, which is not the case for traditional optical measuring apparatus. In terms of data acquisition and transmission, to ensure the simultaneousness of multi-point data collection, a simple bridge deflection monitoring system was initiated. The system consists of a host monitor (PC), distributed inclinometers (CAN slave nodes) and a USB to CAN interface. A matched impedance of 120 is installed in bus end to shun standing waves induced by reflection. Signals are transferred by controller area network (CAN) buses. The CAN controlling local network belongs to field bus, a serial communication network supporting distributive controlling or real-time controlling. The bus-type network topology of such system is illustrated in Fig.1. In this system, the CAN bus is of master-slave communication, that is, the host PC broadcast orders to each sensor via communication protocol in the form of message and then all nodes would collect data simultaneously and transmit the collected data to the host PC per network ID. In terms of data reading at measuring points, an improved Simpson numerical integration scheme is proposed by combining the study on existing dip-to-deflection transformation method with the actual measurement point spacing in the bridge deflection measurement. The tests on bridges unveil that, this scheme could provide precise deflections through unequally spaced measurement points. Furthermore, inclinometer-based bridge deflection measurement method, with its high-precision and easy-maneuverability, is good for short-term bridge monitoring. It could be applied to long-term bridge monitoring, too, thanks to its real-time measurement capability. It has excellent advantages over sense method and associated sense equipment.

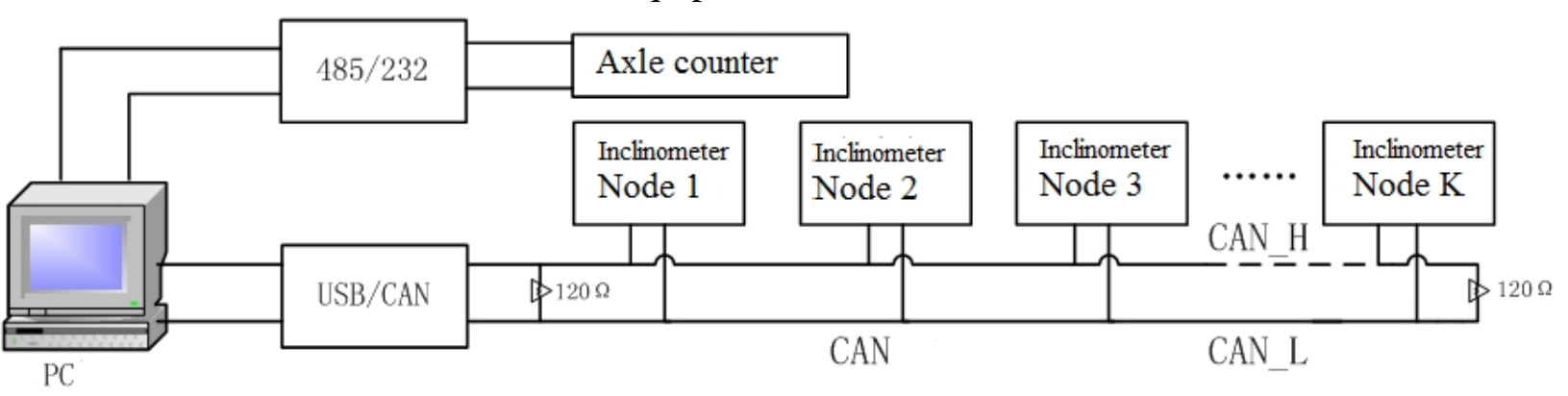

Figure. 1 Multi-point simultaneous data acquisition

On such ground, a fast condition assessment method based on the simple monitoring system is put forward here. Its main idea is that:

I) To save labor and reduce possible impacts on normal traffic, a multi-point high-frequency data acquisition system is developed and the linkage between loading point and bridge deflection is recorded, thus achieving multi-point simultaneous data acquisition.

II) 1 2 loading vehicles drive slowly along the bridge in a given trace.

III) The displacement time-history curve of each measurement point on the bridge structure is real-time collected; and the location of the loading vehicle in the bridge is recorded simultaneously at each time point.

IV) The displacement influence lines of each controlling points are obtained from the measured data above-acquired to produce calculation check coefficient. Evaluation of bridge carrying capacity is implemented using the same method as traditional static loading test.

\section{Quasi-static bridge loading test}

\section{Overview}

The main bridge has 3 spans, to wit, $53 \mathrm{~m}, 85 \mathrm{~m}$, and $53 \mathrm{~m}$. There are also various-sectioned pre-stressed concrete single box continuous box girders. The layout of the bridge deck is $0.5 \mathrm{~m}$ bluster $+16.25 \mathrm{~m}$ vehicle lane $+0.5 \mathrm{~m}$ bluster. Quasi-static loading test is executed on the bridge and the bridge deflection influence line is measured with aforementioned method. The deflections of the 
eight sections equally distributed in the middle span are recorded, i.e. S1 S9. Inclinometers are installed in the sections S10 S13 in the left middle span, except for S1 S9, specifically see Fig. 2.

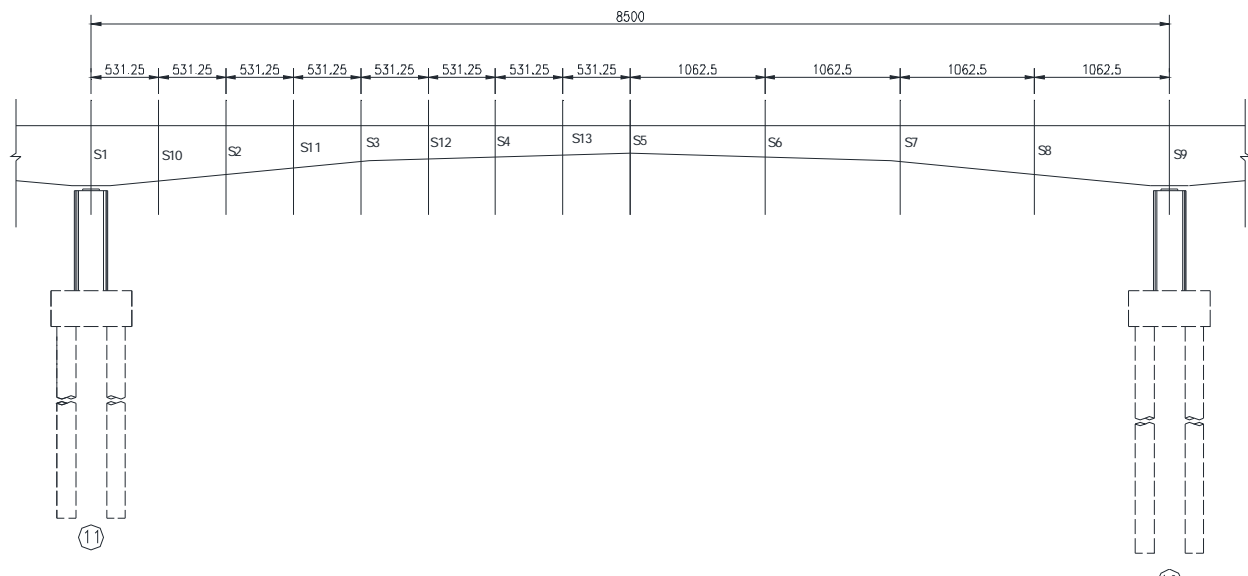

Figure. 2 The location of measured sections

The test employees 2 3D locomotives with a weight of about $300 \mathrm{kN}$ for loading, sees Fig 3 . Table 1 presents some specific parameters of the locomotive.

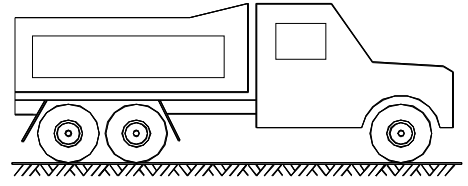

$\lfloor 140 !$

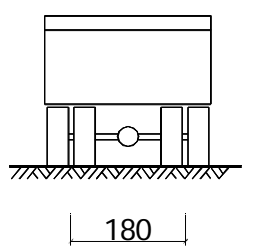

Figure.3 Loading Vehicle

\section{Test result analysis}

During the loading test, a uniform velocity of $5 \mathrm{~km} / \mathrm{h}$ is imposed on the vehicles and they pass the beam-type bridge in different lanes. The inclinometers of the simple monitor system record the deflection at each measurement point as the vehicles pass through the bridge. Meanwhile, on basis of the position data of loading vehicles collected by real-time location, the displacement influence lines under each load case are then obtained by data processing. What is worth mentioning is that, the location devices are installed in the front axis, thus making a time delay of $1.54 / 5 * 3.6=1.1 \mathrm{~s}$, since the distance from the front wheel to the center of vehicle is $154 \mathrm{~cm}$. The following figures illustrate the deflection influence line at $1 / 2$ of the middle span as the loading vehicles pass through lane 1 and land 2 , respectively.

With the analytical result of quasi-static loading test taken advantage of, the deflection at the measurement points as the load locates in any place of main bridge could be inferred. To further verify the test result, a static loading test was conducted. The two vehicles were placed in the lane 1 and lane 2 in the mid-span of the middle span and the corresponding deflection was intentionally measured with a static level gauge, which is $2.97 \mathrm{~mm}$. Fig. 4 reads, at the position of $(53+85 / 2)$ $=95.5 \mathrm{~m}$, associated deflections are $1.53 \mathrm{~mm}$ and $1.42 \mathrm{~mm}$, respectively. The principle of superposition gives the result of $2.95 \mathrm{~mm}$, a result very close to that given by the static loading test. Hence, the quasi-static loading test could serve as a substitute of normal static loading test in such case. 


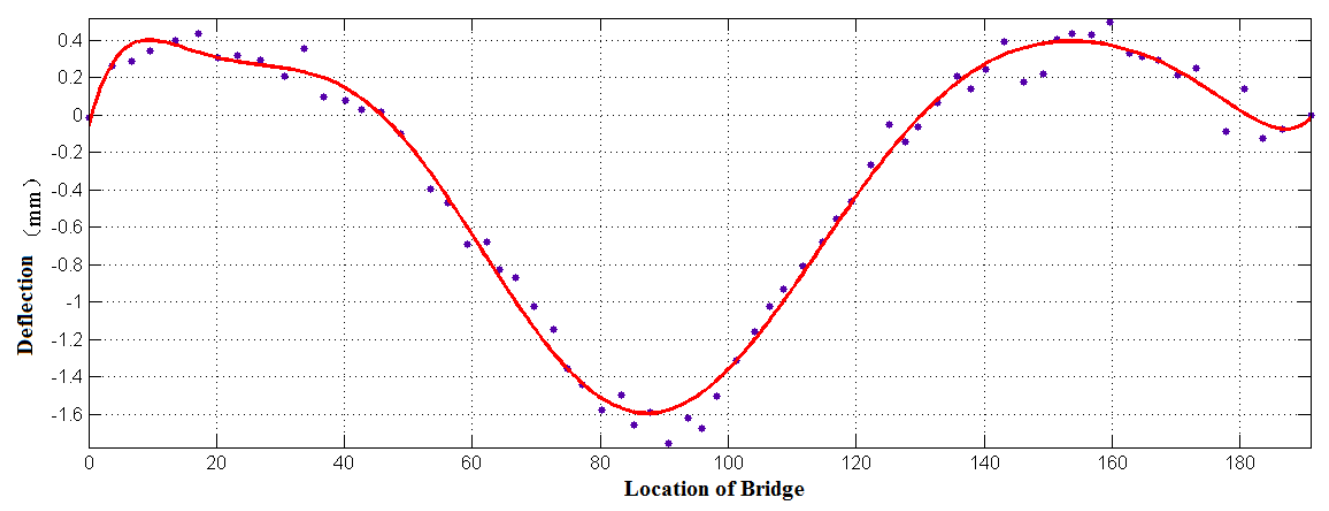

Figure. 4 Mid-span deflection influence lines under different load cases

\section{Conclusion}

(1) The construction of the simple monitoring system, which combines up-to-date sense technologies with the development of data acquisition software, lay a foundation of the application of the fast condition assessment method. The quasi-static loading test could take the replace of traditional loading test, saving labor and material while reducing the possible influence on traffic.

(2) In this paper, it is the mid-span of the middle span of the main-bridge that is selected for the verification. The result analysis disclosed the accuracy and reliability of the mid-span deflection measured with the proposed method. Similarly, further researches could focus on the application and the popularization of the proposed method in different types of bridge and in different parts of a bridge.

(3) The fast condition assessment method based on simple monitoring system could assess bridge conditions and evaluate the carrying capacity of bridge, which could provide a better prediction of potential damage in the long-term service life of the bridge and could ensure the health of the bridge and its functionability in promoting local economy.

\section{Reference}

[1] Zhou J.T., Hao Y..Study of Bridge's Practical Influence Line from Recurrence Iterative Testing and Its Use in Bridge's Bearing Capacity Evaluation [J] , 2006 , 23 ( 6 ) : 81-84.

[2] Li Y.H. Preliminary study on the loading bearing capacity of middle-span and short-span beam-type bridge based on quasi-static generalized influence line [D], Chongqing Jiaotong University , 2009

[3] Liao J.B. , Tang G.W. et al. Research on Application of Measured Quasistatic Generalized Influence Line in Static Load Test for Bridge Structure [J] , Technology of Highway and Transport , 2009 , ( 6 ) : 65-68.

[4] Zhang Y.F.,Duan H.J,Ma Z.G. Fast Appraisal for Girder Bridge Condition Based on Quasi Stationary Load Test [J]. Modern Transportation Technology, 2009, 05:21-24

[5] Filipe Cavadas, IanF.C.Smith, Joaquim Figueiras , Damage detection using data-driven methods applied to moving-load responses [J] , Mechanical Systems and Signal Processing , 2013 , 39 : 409-425. 